\title{
Apoptotic Cell-Mimetic Polymers for Anti-Inflammatory Therapy
}

\author{
Mitsuhiro Ebara ${ }^{1,2,3, *}$ \\ ${ }^{1}$ International Center for Materials Nanoarchitectonics (WPI-MANA), National Institute for Materials Science (NIMS), ${ }^{2}$ Graduate School \\ of Pure and Applied Sciences, University of Tsukuba, Tsukuba, ${ }^{3}$ Graduate School of Industrial Science and Technology, Tokyo University \\ of Science, Tokyo, Japan
}

The field of biomaterials has seen a strong rejuvenation due to the new potential to modulate immune system in our body. This special class of materials is called "immunomodulatory biomaterials". Generally, three fundamental strategies are followed in the design of immunomodulatory biomaterials: (1) immuno-inert biomaterials, (2) immuno-activating biomaterials, and (3) immuno-tolerant biomaterials. While many applications of immuno-inert biomaterials such as biocompatible medical implants have been already proposed in the past decades, the ability to engineer biological activity into synthetic materials greatly increases the number of their potential uses and improves their performance in more traditional applications. The major focus of researchers is now set on developing immuno-tolerant biomaterials for anti-inflammatory therapies. In this review, we therefore introduce recent developments of immuno-tolerant biomaterials. Especially we introduce an apoptotic cell membraneinspired polymer and its post-inflammatory effects on immune cells in this article.

\section{Key Words: Apoptosis; Phosphatidylserines; Anti-Inflammatory Agents; Immunomodulation; Biocompatible Materials}

This is an Open Access article distributed under the terms of the Creative Commons Attribution Non-Commercial License (http://creativecommons.org/licenses/by-nc/4.0) which permits unrestricted non-commercial use, distribution, and reproduction in any medium, provided the original work is properly cited.

\section{Article History:}

Received October 4, 2018

Revised November 28, 2018

Accepted December 13, 2018

\section{Corresponding Author: Mitsuhiro Ebara International Center for Materials Nanoarchitectonics (WPI-MANA), National Institute for Materials Science (NIMS), 1-1 Namiki, Tsukuba, Ibaraki 305-0044, Japan \\ Tel: $+81-29-860-4775$ \\ Fax: +81-29-860-4708 \\ E-mail:EBARA.Mitsuhiro@nims.go.jp}

\section{INTRODUCTION}

\section{History of immuno-inert biomaterials}

Biomaterials have been historically developed with the aim to transplant then into the human body as substitutes for damaged tissue or a biological function. ${ }^{1,2}$ Biomaterials may be metals, ceramics, polymers, or even living cells and tissue. They can be used as surface coatings, fibers, films, or particles for use in biomedical products such as heart valves, hip joint replacements, dental implants, or drug delivery carriers. The first generation of biomaterials was developed under the concept of immune evasion because the biggest challenge of "foreign objects" has been the action of the immune system. Therefore, it was preferable that they be 'inert' and not interact with the biology of the host organism such as proteins, lipids, nucleic acids, sugars, and amino acids. One of the most successful approaches to produce bio-inert materials has been the used of polyethylene glycol (PEG). ${ }^{3}$ For example, covalent attachment of PEG to proteins or drugs called "PEGylation" has been shown to improve the safety and efficiency, and many
PEGylated pharmaceuticals are currently on the market. This technology can be also used as a means to design anti-fouling surfaces. Grafting PEG to solid surfaces significantly reduces protein adsorption and cell adhesion. ${ }^{4-6}$ In addition to PEG, zwitterionic polymers have recently been extensively developed for anti-fouling purpose. ${ }^{7-9}$ Among them, one of the cell membrane lipids, phosphatidylcoline (PtdCho)-inspired polymers such as 2-methacryloyoxyethyl phosphorylcholine (MPS) have been used in various medical devices. ${ }^{10-12}$ These polymers have extended the applications of biomaterials from traditional implants to biosensing, prodrug carriers, subcellular bioimaging, and cell manipulation.

\section{Challenges of immuno-modulating biomaterials}

Since studies of bio-inert biomaterials are already well advanced, many researchers have recently switched their focus from bio-inert to bio-modulating materials that promote or inhibit immune responses. However, controlling the immune system with biomaterials is still a challenge due to their methodological complexity. For example, an- 
ti-tumor activity is expected when immune systems are activated, while excess activation can cause unexpected symptoms such as allergies (Fig. 1). On the other hand, suppressing the immune system lowers inflammation, but also leads to diminished resistance against infections. Therefore, to obtain immunotherapy with a biomaterial, it is necessary to perfectly understand the biological reactions induced by implanted materials and to adequately design the shape, physical properties, and chemical prop- erties of the material. Fig. 2 shows three types of biomaterials according to their association with the immune system. The first category is called "immuno-inert biomaterials" as described above. The second type is called "immuno-activating biomaterials" designed to exhibit anti-tumor and drug-responsive properties. The third type is known as "immuno-tolerant biomaterials" which suppress and modulate unnecessary uncontrollable inflammation and inhibit rejection responses.
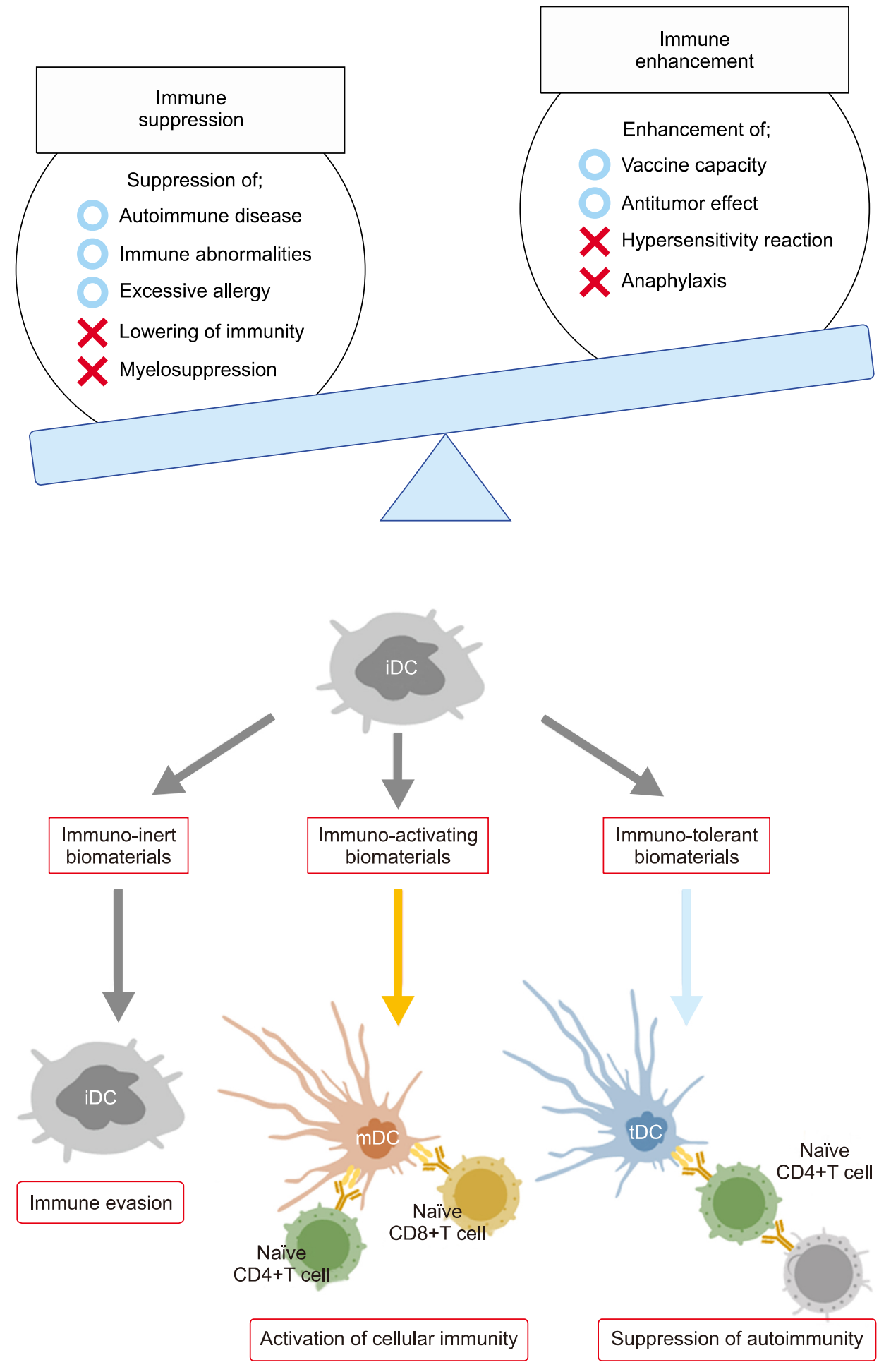

Fig. 1. Improvement of immunity. The trade-off relationship between immunoactivation and immuno-suppression.

Fig. 2. Biological reactions and expected effects of immuno-inert, immuno-activating, and immuno-tolerant biomaterials. iDC: immature dendritic cell, $\mathrm{mDC}$ : mature dendritic cell, tDC: tolerogenic dendritic cell. 


\section{IMIMUNE-ACTIVATING BIOMATERIALS}

\section{Adjuvant materials}

As mentioned above, much effort has been previously made to design biomaterials to minimize the host's immune responses against implanted materials. However, biomaterials can also be designed to activate the host's immune responses and/or provide therapeutic effects. The first application of this concept was the use of non-biological adjuvant materials such as $\gamma$-polyglutamic acid $(\gamma$ -PGA), ${ }^{13}$ poly (lactic-co-glycolic acid) (PLGA), ${ }^{14}$ or poly (E-caprolactone) (PCL). ${ }^{15}$ These biodegradable materials can increase the host's immune response to vaccines. New types of $\mathrm{pH}$-responsive nanoparticles have also been developed because the degradation rate for such biodegradable polymers is not fast enough for effective antigen deliveries. ${ }^{16}$ The nanoparticles with $\mathrm{pH}$-cleavable crosslinkers are rapidly hydrolyzed under lysosomal acidic conditions (pH 5) and release antigens into dendritic cells (DCs). By contrast, Stayton and co-workers proposed antigen delivery strategies that mimic virus infection mechanisms. They focused on polypropylic acid (PPAA), which has a $\mathrm{p} K_{\mathrm{a}}$ around $\mathrm{pH}$ 6.0-6.5, making it hydrophilic at a biological $\mathrm{pH}$ and dramatically hydrophobic at endosomal $\mathrm{pH}$ due to the protonation of carboxyl groups along its backbone. ${ }^{17}$ Due to the polymer phase transition, PPAA disrupted lipid bilayer membranes and antigens tethered to PPAA by disulfide bonds were released under endosomal reductive conditions.

\section{Hemagglutinating virus of Japan-envelope (HVJ-E)}

Anti-tumor immunity can be also induced by viral envelopes such as Hemagglutinating Virus of Japan-envelope (HVJ-E) ${ }^{18}$ HVJ-E is a purified product prepared through the complete inactivation of the genome in HVJ by UV irradiation. HVJ-E was originally developed as a novel vector for plasmid DNA, peptides, and drugs because of the fusion proteins of the HVJ such as $\mathrm{HN}$ and $\mathrm{F}$ are retained after inactivation. Recently, Kaneda and colleagues have reported the tumor-suppressing ability of the inactivated, replication-defective HVJ-E itself. ${ }^{19}$ HVJ-E alone induced tumor-specific antitumor immunity by eliciting IL-6 production in dendritic cells (DCs) and eradicated 60\% to $80 \%$ of tumors growing in mice without exogenous gene expression. Thus, HVJ-E has recently attracted a lot of attention as a new type of therapeutic material for cancer immunotherapy. However, one of the greatest challenges for viral-based vectors is that they tend to fuse with virtually all cells and induce hemagglutination in the bloodstream. Therefore, it has been required to increase the tumor-targeting ability as well as reduce nonspecific biding. To achieve this, the chemical modification of the viral vector is one of the most direct approaches. For example, surface-modified adenoviral vectors (Ads) with a multivalent reactive poly [N- (2-hydroxypropyl) methacrylamide] (PHPMA)-based copolymer has been shown successfully to shield from recognition by antibodies. ${ }^{20}$ The authors have fashioned HVJ-E with hyaluronic acid (HA) to act as a ligand for CD44 to improve its anti-tumor activity. ${ }^{21,22}$ The functionalized HVJ-E is expected to improve the retention time in the blood stream by inhibiting interactions between HVJ-E and red blood cells. Also, the HA layer can be selectively degraded in cancer tissues by hyaluronan-targeting enzymes. A layer-by-layer (LbL) assembly has been employed for the decoration of the HVJ-E. We have succeeded in forming $\mathrm{HA} / \mathrm{glycol}$ chitosan (GC) multilayers on HVJ-E for up to six assembly cycles. The outermost HA layer was selectively degraded by hyaluronidase. The HA/GC-coated HVJ-E can be thought of as a potential bomb for cancer immunotherapy because of its ability to target CD44 while preventing nonspecific binding in the bloodstream.

\section{IMMUNE-SUPPRESSING BIOMATERIALS}

\section{Induction of immune tolerance}

In addition to immune-activating biomaterials, researchers have been recently developing immuno-tolerant or suppressing materials for the treatment of transplant rejections and autoimmune diseases. In general, the immune system effectively eliminates foreign substances such as pathogenic microorganisms, but does not respond to self-biomolecules, symbiotic microorganisms, or environmental substances. However, in autoimmune diseases, the immune system misidentifies some molecules as foreign and elicits a response against them. For example, chronic inflammatory disorders such as inflammatory bowel disease, allergic reactions, cancer, obesity, diabetes, collagen disease, and Alzheimer's disease are all caused by the impaired maintenance of immune homeostasis. To induce immune tolerance, several strategies have been developed by using various biomaterials for the delivery of adjuvants, antigens, and drugs. For example, PLGA particles that contained costimulatory anti-sense oligonucleotides for DCs and T cells successfully inhibited immune responses. ${ }^{23}$ In another example, PLGA particles modified with anti-DEC205 antibodies, anti-CD1c antibodies, and P-2 peptides were also developed. ${ }^{24}$ These examples show the potential application of targeting PLGA particles to maintain immunological tolerance.

However, previous immune-suppressive biomaterials as mentioned above simply operate as delivery carriers. In other words, the materials themselves do not have any anti-inflammatory properties. However, in recent years, material-based immuno-suppressive systems have been developed, and a few specific examples will be introduced here. Stable, organic free radicals such as 2, 2, 6, 6-tetramethylpiperidine 1-oxyl (TEMPO) are often used as catalysts for oxidation reactions in organic synthesis and can act as radical scavengers that detect radical generation reacting systems. Yoshitomi et al. ${ }^{25}$ focused on the radical scavenging ability of TEMPO for removing reactive oxygen species (ROS) at inflammatory regions. Normally, large amounts of ROS are produced during inflammation, and exposure to this oxidative stress can cause various diseases such as 
arteriosclerosis. However, reactive oxygen must be selectively removed as it plays an important role during intracellular aerobic respiration in mitochondria. They succeeded in improving the cognitive function of mice that model Alzheimer disease by orally administering TEMPOfunctionalized $\mathrm{PEG}{ }^{26}$

\section{Apoptotic cell-inspired biomaterials}

Ideally, biomaterials should mimic the living tissue or biomolecules from a mechanical, chemical, biological, and functional point of view. As mentioned in the introduction, one of the most successful biocompatible polymers, MPC polymer, was developed by learning from mammalian cell membrane lipid PtdCho. MPC polymers have been extensively applied to various medical devices, including an artificial joint (Aquala ${ }^{\circledR}$, Japan Medical Materials), cardiovascular stent (Endeavor ${ }^{\circledR}:$ Medtronic), left ventricular assist device (EVAHEART ${ }^{\circledR}$, Sun Medical), oxygenator (PrimO2x ${ }^{\circledR}$ : Sorin), and soft contact lenses (Proclear ${ }^{\circledR}$ : Cooper Vision). Therefore, we discuss the potential to create a novel immune-suppressing biomaterial by focusing on the lipid membrane in the last section.

In general, cell membranes have a lipid bilayer constructed by phospholipids such as electrically neutral sphingomyelin (Sph) and PtdCho that occupy the outside membrane and anionically charged phosphatidylethanolamine (PtdEA), PtdSer, and phosphatidylinositol (PtdIno) that are located on the cytosol side. ${ }^{27}$ Cell membranes keep this heterogeneous structure by active transportation at inner sites by scramblase, and the cytoskeleton is therefore stabilized by anionic phospholipids. However, apoptosis induces the destruction of these highly maintained asymmetrical structures, forcing PtdSer to be exposed on the surface of apoptotic cell membranes, which are then recognized by immune cells (Fig. 3) ${ }^{28}$ Apoptosis is a genetically programmed cell death mechanism and is necessary to regulate embolic development and maintain homeostasis. Many studies also revealed that PtdSer acts as a trigger molecule for these anti-inflammatory effects. ${ }^{29}$ Voll et al..$^{30}$ reported that apoptosis induced leukocytes to downregulate inflammatory cytokine production and upregulate anti-inflammatory cytokines for the production of immune cells. Several therapeutic effects of PtdSer liposomes have been also reported for chronic heart failure, ${ }^{31}$ Alzheimer's disease ${ }^{32}$ and osteoporosis. ${ }^{33}$ Table 1 summarizes the recent progress of anti-inflammatory therapies using apoptotic cells and PtdSer liposomes. ${ }^{30-37}$

We have recently designed apoptotic cell membrane-inspired polymers which have a phosphoryl serine (PS) group because PtdSer mimetic polymers are very promising materials for anti-inflammatory therapies (Fig. 4). ${ }^{38,39}$ However, the synthesis of PtdSer-mimetic polymers has been diffi-

TABLE 1. Anti-inflammatory effects using apoptotic cells and PtdSer liposomes

\begin{tabular}{llc}
\hline \multicolumn{1}{c}{ Samples } & Anti-inflammatory effects & Ref. \\
\hline Apoptotic cells & IL-10 $\uparrow$, TNF- $\alpha \downarrow$ & 30 \\
Apoptotic cells & TGF- $\beta 1 \uparrow$ & 34 \\
Apoptotic cells & TGF- $\beta 1 \uparrow$, IL-10 $\uparrow$ & 35 \\
Apoptotic cells & IL-12 $\downarrow$, TNF- $\alpha \downarrow$, IFN- $\gamma \downarrow$ & 36 \\
Liposomes & IL-10 $\uparrow$, TNF- $\alpha \downarrow$ & 31 \\
Liposomes & TNF- $\alpha \downarrow$, NO $\downarrow$ & 32 \\
Liposomes & TGF- $\beta 1 \uparrow$, PEG $2 \uparrow$ & 33 \\
Liposomes & IL-10 $\uparrow$, IL-1b $\downarrow$ & 37 \\
\hline
\end{tabular}<smiles>CCC(=O)NC(COP(=O)(O)OCC[N+](C)(C)C)C(O)C=C(C)C</smiles>

Sph

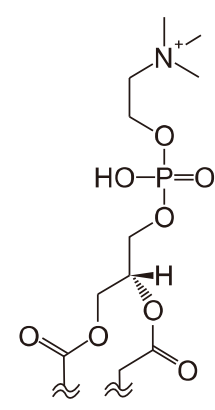

PtdCho

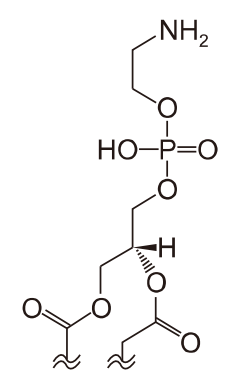

PtdEA<smiles>CC(=O)OC[C@@H](COP(=O)(O)OCC(N)C(=O)O)OC(C)=O</smiles>

PtdSer

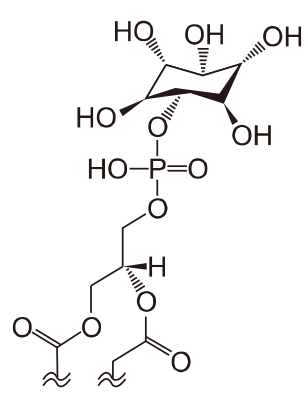

Ptdlno

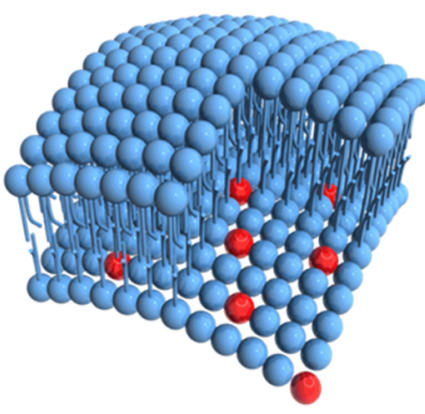

Normal cell

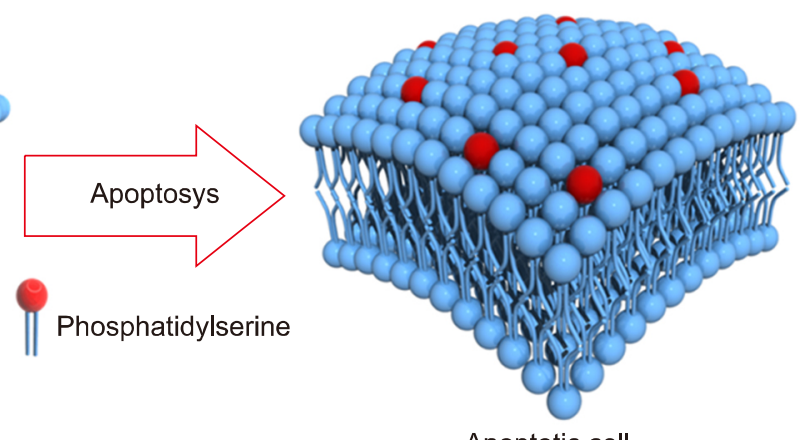

Apoptotic cell
Fig. 3. Major phospholipids constituting the cell membrane and collapse of asymmetrical distribution of the phospholipid bilayer due to the progression of apoptosis. Sph: sphingomyelin, PtdCho: phosphatidylcholine, PtdEA: phosphatidylethanolamine, PtdSer: phosphatidylserine, PtdIno: phosphatidylinositol. 


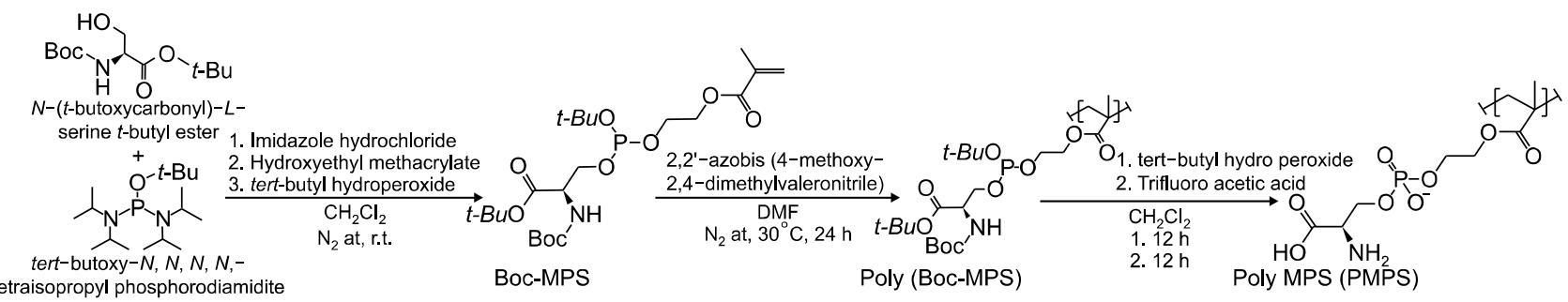

FiG. 4. Synthesis of an apoptotic cell membrane-mimetic polymer via phosphoramidite chemistry.

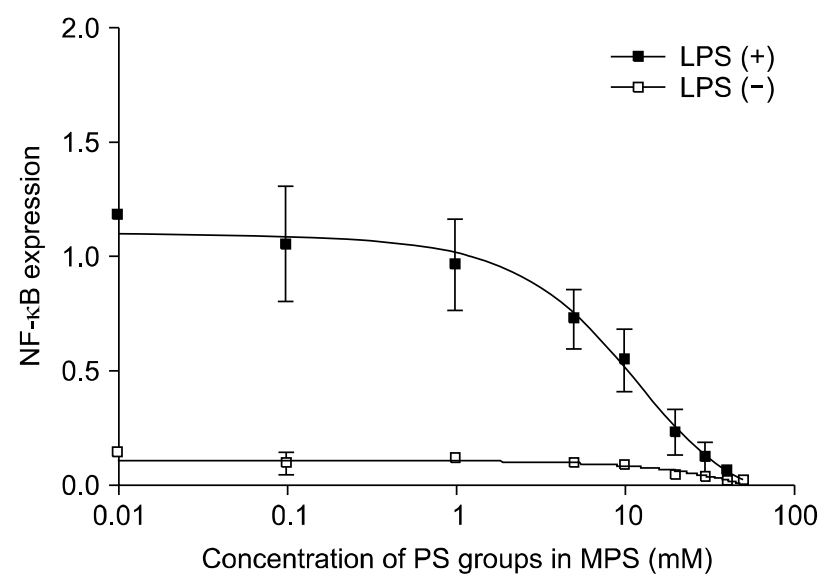

FiG. 5. Effects of poly (MPS) on the NF- $\mathrm{KB}$ expression in LPSstimulated macrophages.

cult as serine has two reactive groups (the amino- and carboxy-groups) that limit the effective and selective modification routes. Therefore, we used a phosphoramidite method which is commonly used in solid phase syntheses of DNA. The advantage of using this reaction is its high selectivity under mild conditions when forming a phosphate ester between two hydroxyl groups. ${ }^{39}$ First, we synthesized the methacryloyloxyethyl phosphorylserine (MPS) monomer using 2-hydroxyethyl methacrylate, $N$ - $\alpha$-(t-butoxycarbonyl)- $L$-serine $t$-butyl ester, and $t$-butyl tetraisopropylphosphorodiamidite. Next, poly (MPS) was obtained by free radical polymerization and then deprotection and oxidation of the PtdSer using $t$-butyl peroxide and trifluoroacetic acid. We applied poly (MPS) to lipopolysaccharide (LPS)-activated RAW 264.7 macrophages to investigate the immune-suppressive effects. Amoeboid cell extensions of macrophages in LPS stimulated conditions were inhibited in the poly (MPS)-treated groups. In addition, the effective suppression of NF- $\mathrm{kB}$ in macrophages was observed over $10 \mathrm{mM}$ of PS groups (Fig. 5). ${ }^{38}$ To investigate how macrophages recognize poly (MPS), the localization of rhodamine-modified poly (MPS) on macrophages was studied. A homogeneous distribution of the poly (MPS) in the cytoplasm was observed. By calculating the up-taken amount of poly (MPS) in the cell, we found that $50 \mathrm{pg}$ or more of poly (MPS) within each macrophage is necessary to inhibit inflammatory behavior (Fig. 6). Furthermore, we investigated the time-dependent immunosuppression induction of poly (MPS). The NF-kB expression was inhibited

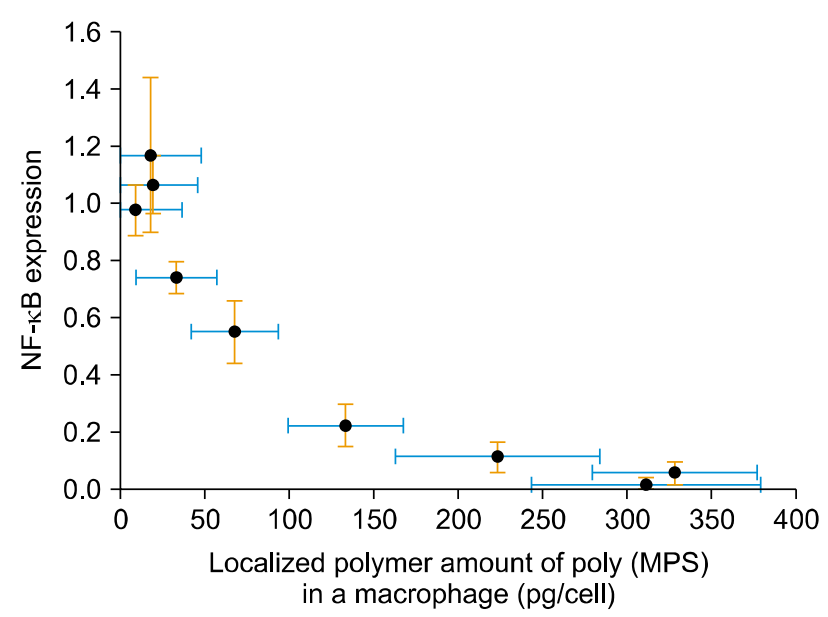

FiG. 6. NF- $-\mathrm{KB}$ expression against localized poly (MPS) amount in the cytosol in RAW macrophages.

after $3 \mathrm{~h}$ incubating, indicating that a minimum time for immunosuppression is $3 \mathrm{~h}$. The findings in this study are expected to open new doors in developing advanced immuno-suppressive agents and materials for anti-inflammatory therapies such as autoimmune diseases.

\section{CONCLUSION}

This review article summarizes recent research examples of immuno-modulating biomaterials and systems. These materials are classified into three categories: (1) immuno-inert, (2) immuno-activating, and (3) immune-tolerant biomaterials. Particularly, we mainly focused on the immuno-tolerant biomaterials in this article. In previous immuno-tolerant biomaterial systems, the materials themselves did not have any anti-inflammatory properties. However, the ability to engineer biological activity into synthetic materials has enabled the design of biomaterials which harness the host's immune responses and provide therapeutic effects. One of the examples is apoptotic cellmembrane mimetic polymers. Advantages of using such polymeric materials for anti-inflammatory therapy is cost-effectiveness as it is inexpensive compared to molecular targeted drugs. However, controlling the immune system is still a challenge due to its methodological complexity. For example, excess activation of the immune system may cause unexpected symptoms such as allergies, while suppressing of the immune system also leads to diminished re- 
sistance against infections. Therefore, to obtain immunotherapy with a biomaterial, it is necessary to design the shape, physical properties, and chemical properties of the material adequately. In the future, biomaterials will assume an even greater role in anti-inflammatory therapies through biologically inspired design.

\section{ACKNOWLEDGEMENTS}

This research was funded by JSPS KAKENHI (Grant Number 264086 and 26750152).

\section{CONFLICT OF INTEREST STATEMENT}

None declared.

\section{REFERENCES}

1. Ratner BD, Bryant SJ. Biomaterials: where we have been and where we are going. Annu Rev Biomed Eng 2004;6:41-75.

2. Huebsch N, Mooney DJ. Inspiration and application in the evolution of biomaterials. Nature 2009;462:426-32.

3. Abuchowski A, McCoy JR, Palczuk NC, van Es T, Davis FF. Effect of covalent attachment of polyethylene glycol on immunogenicity and circulating life of bovine liver catalase. J Biol Chem 1977;252: 3582-6.

4. Uchida K, Hoshino Y, Tamura A, Yoshimoto K, Kojima S, Yamashita K, et al. Creation of a mixed poly(ethylene glycol) tethered-chain surface for preventing the nonspecific adsorption of proteins and peptides. Biointerphases 2007;2:126-30.

5. Alcantar NA, Aydil ES, Israelachvili JN. Polyethylene glycol-coated biocompatible surfaces. J Biomed Mater Res 2000;51: 343-51.

6. Leckband D, Sheth S, Halperin A. Grafted poly(ethylene oxide) brushes as nonfouling surface coatings. J Biomater Sci Polym Ed 1999;10:1125-47.

7. Ladd J, Zhang Z, Chen S, Hower JC, Jiang S. Zwitterionic polymers exhibiting high resistance to nonspecific protein adsorption from human serum and plasma. Biomacromolecules 2008;9: 1357-61.

8. Li A, Luehmann HP, Sun G, Samarajeewa S, Zou J, Zhang S, et al. Synthesis and in vivo pharmacokinetic evaluation of degradable shell cross-linked polymer nanoparticles with poly(carboxybetaine) versus poly(ethylene glycol) surface-grafted coatings. ACS Nano 2012;6:8970-82.

9. Zheng L, Sundaram HS, Wei Z, Li C, Yuan Z. Applications of zwitterionic polymers. React Func Polym 2017;118:51-61.

10. Ishihara K, Aragaki R, Ueda T, Watenabe A, Nakabayashi N. Reduced thrombogenicity of polymers having phospholipid polar groups. J Biomed Mater Res 1990;24:1069-77.

11. Goda T, Ishihara K, Miyahara Y. Critical update on 2-methacryloyloxyethyl phosphorylcholine (MPC) polymer science. J Appl Polym Sci 2015;132:41766.

12. Mu M, Konno T, Ishihara K. Spontaneous hydrogel formation through hydrophobic interactions in an ABA-type block copolymer composed of poly(2-methacryloyloxyethyl phosphorylcholine) and poly(n-butyl methacrylate) segments. MRS Advances
2018;3:1691-6.

13. Nilsson JS, Broos S, Akagi T, Akashi M, Hermansson A, Cayé-Thomasen P, et al. Amphiphilic $\gamma$-PGA nanoparticles administered on rat middle ear mucosa produce adjuvant-like immunostimulation in vivo. Acta Otolaryngol 2014;134:1034-41.

14. Jiang W, Gupta RK, Deshpande MC, Schwendeman SP. Biodegradable poly(lactic-co-glycolic acid) microparticles for injectable delivery of vaccine antigens. Adv Drug Deliv Rev 2005;57: 391-410.

15. Benoit MA, Baras B, Gillard J. Preparation and characterization of protein-loaded poly(epsilon-caprolactone) microparticles for oral vaccine delivery. Int J Pharm 1999;184:73-84.

16. Kwon YJ, James E, Shastri N, Fréchet JM. In vivo targeting of dendritic cells for activation of cellular immunity using vaccine carriers based on $\mathrm{pH}$-responsive microparticles. Proc Natl Acad Sci U S A 2005;102:18264-8.

17. Murthy N, Robichaud JR, Tirrell DA, Stayton PS, Hoffman AS. The design and synthesis of polymers for eukaryotic membrane disruption. J Control Release 1999;61:137-43.

18. Kaneda Y, Yamamoto S, Nakajima T. Development of HVJ envelope vector and its application to gene therapy. Adv Genet 2005; 53:307-32.

19. Kurooka M, Kaneda Y. Inactivated Sendai virus particles eradicate tumors by inducing immune responses through blocking regulatory T cells. Cancer Res 2007;67:227-36.

20. Fisher KD, Stallwood Y, Green NK, Ulbrich K, Mautner V, Seymour LW. Polymer-coated adenovirus permits efficient retargeting and evades neutralising antibodies. Gene Ther 2001;8: 341-8.

21. Okada T, Uto K, Sasai M, Lee CM, Ebara M, Aoyagi T. Nano-decoration of the Hemagglutinating Virus of Japan envelope (HVJ-E) using a layer-by-layer assembly technique. Langmuir 2013;29: 7384-92.

22. Okada T, Uto K, Aoyagi T, Ebara M. A biomimetic approach to hormone resistant prostate cancer cell isolation using inactivated Sendai virus (HVJ-E). Biomater Sci 2016;4:96-103.

23. Phillips B, Nylander K, Harnaha J, Machen J, Lakomy R, Styche A, et al. A microsphere-based vaccine prevents and reverses new-onset autoimmune diabetes. Diabetes 2008;57:1544-55.

24. Lewis JS, Zaveri TD, Crooks CP 2nd, Keselowsky BG. Microparparticle surface modifications targeting dendritic cells for non-activating applications. Biomaterials 2012;33:7221-32.

25. Yoshitomi T, Yamaguchi Y, Kikuchi A, Nagasaki Y. Creation of a blood-compatible surface: a novel strategy for suppressing blood activation and coagulation using a nitroxide radical-containing polymer with reactive oxygen species scavenging activity. Acta Biomater 2012;8:1323-9.

26. Boonruamkaew P, Chonpathompikunlert P, Vong LB, Sakaue S, Tomidokoro Y, Ishii K, et al. Chronic treatment with a smart antioxidative nanoparticle for inhibition of amyloid plaque propagation in Tg2576 mouse model of Alzheimer's disease. Sci Rep 2017;7:3785.

27. Verkleij AJ, Post JA. Membrane phospholipid asymmetry and signal transduction. J Membr Biol 2000;178:1-10.

28. Somersan S, Bhardwaj N. Tethering and tickling: a new role for the phosphatidylserine receptor. J Cell Biol 2001;155:501-4.

29. Gaipl US, Beyer TD, Baumann I, Voll RE, Stach CM, Heyder P, 
et al. Exposure of anionic phospholipids serves as anti-inflammatory and immunosuppressive signal--implications for antiphospholipid syndrome and systemic lupus erythematosus. Immunobiology 2003;207:73-81.

30. Voll RE, Herrmann M, Roth EA, Stach C, Kalden JR, Girkontaite I. Immunosuppressive effects of apoptotic cells. Nature 1997;390: 350-1.

31. Harel-Adar T, Ben Mordechai T, Amsalem Y, Feinberg MS, Leor J, Cohen S. Modulation of cardiac macrophages by phosphatidylserine-presenting liposomes improves infarct repair. Proc Natl Acad Sci U S A 2011;108:1827-32.

32. Hashioka S, Han YH, Fujii S, Kato T, Monji A, Utsumi H, et al. Phosphatidylserine and phosphatidylcholine-containing liposomes inhibit amyloid beta and interferon-gamma-induced microglial activation. Free Radic Biol Med 2007;42:945-54.

33. Wu Z, Ma HM, Kukita T, Nakanishi Y, Nakanishi H. Phosphatidylserine-containing liposomes inhibit the differentiation of osteoclasts and trabecular bone loss. J Immunol 2010;184:3191-201.

34. Huynh ML, Fadok VA, Henson PM. Phosphatidylserine-depend- ent ingestion of apoptotic cells promotes TGF-beta1 secretion and the resolution of inflammation. J Clin Invest 2002;109:41-50.

35. Thum T, Bauersachs J, Poole-Wilson PA, Volk HD, Anker SD. The dying stem cell hypothesis: immune modulation as a novel mechanism for progenitor cell therapy in cardiac muscle. J Am Coll Cardiol 2005;46:1799-802.

36. Ren Y, Xie Y, Jiang G, Fan J, Yeung J, Li W, et al. Apoptotic cells protect mice against lipopolysaccharide-induced shock. J Immunol 2008;180:4978-85.

37. Nolan Y, Martin D, Campbell VA, Lynch MA. Evidence of a protective effect of phosphatidylserine-containing liposomes on lipopolysaccharide-induced impairment of long-term potentiation in the rat hippocampus. J Neuroimmunol 2004;151:12-23.

38. Nakagawa Y, Saitou A, Aoyagi T, Naito M, Ebara M. Apoptotic cell membrane-inspired polymer for immunosuppression. ACS Macro Lett 2017;6:1020-4.

39. Nakagawa Y, Saitou A, Aoyagi T, Ebara M. Rational design of anti-inflammatory polymers inspired by apoptotic cell death using phosphoramidite chemistry. Polymer 2018;134:85-93. 